\title{
Highlights of the Year
}

\author{
Physics picks its favorite stories from 2020
}

$\Gamma$ here is no denying that the raging pandemic shaped the world of physics in 2020, shifting research priorities, hindering in-person meetings, disrupting supply chains, and leaving a psychological toll on students, teachers, and researchers. Many physicists from around the world shared with us their experiences coping with the pandemic. Yet physics research in all fields marched on and kept delivering surprises and breakthroughs. The selection of our favorite stories of the year clearly reflects the resilience and ingenuity of the physics community.

Wishing everyone a safe and bright 2021.

-The Editors

\section{Physicists Tackle COVID-19}

The pandemic has been a challenge to the whole planet. To do their part, physicists from diverse fields lent their expertise to efforts like simulating disease spread and designing medical equipment (see Research News: Forecasting COVID-19's Trajectory, Physicists Design Emergency Ventilators, and A Bead-Based Test for COVID-19). A team helped in modeling the effects of stay-at-home orders, which influenced the government's response in the state of Illinois (see Viewpoint:

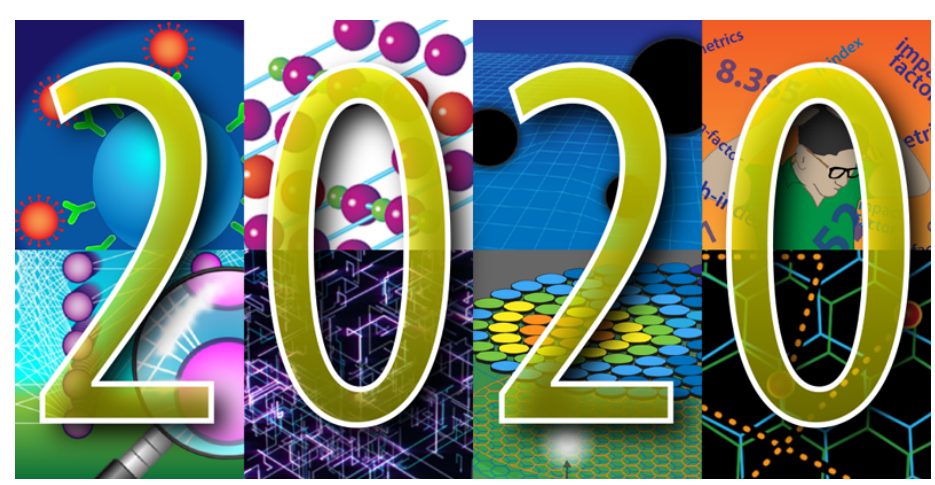

Credit: APS/Alan Stonebraker
The Uncertain Future in How a Virus Spreads). Unfortunately, widespread quarantine-flaunting behavior made their statistical predictions overly optimistic-a realization that could help researchers build more realistic models for fighting the virus.

\section{Healthy Minds}

This year, Physics significantly expanded its Opinion section-in which researchers share their views on issues impacting the physics community. One of our favorite Opinion pieces was a trio of essays by mental health advocates (see Opinon: Speaking up for Mental Health). The essays covered the importance of candid conversations in breaking the stigma surrounding mental health struggles, the need for support communities in physics, and the burden that publication metrics place on academics. "Now, more than ever, when isolation and racial injustice are at the forefront of our collective consciousness, we need... to remember we are not alone," wrote Andrea Welsh.

\section{Dark Matter Inklings?}

An unexplained signal from the XENON1T experiment bears the palm for the year's biggest shakeup in dark matter research (see Viewpoint: Dark Matter Detector Delivers Enigmatic Signal). The flashes of light seen by the detector could be harbingers of new physics, but the signals haven't yet reached the "5-sigma" bar for a discovery, nor have the researchers fully ruled out a mundane origin of the signal. Still, theorists have reacted swiftly to the news, exploring ideas that include exotic neutrinos and different types of dark matter particles (see Synopsis: Theorists React to Potential Signal in Dark Matter Detector).

\section{Quantum Prospects}

Several research groups have tested quantum networks tens of miles in size, but to realize the larger networks needed for a quantum internet, they will need a quantum repeater (see Research News: The Key Device Needed for a Quantum Internet). This specialized piece of equipment reads and 
re-emits quantum states without destroying their superpositions, but more research is needed to make quantum repeaters ripe for applications. Besides this hardware component, other developments-in society as well as in technology-will be crucial for meeting our quantum potential, as US quantum technology leaders discussed in a series of essays (see Opinion: Ingredients for a Quantum Future).

\section{LIGO and Virgo Spy Their Heaviest Merger Yet}

Just when gravitational-wave sightings of black hole mergers seemed to have become routine, the LIGO and Virgo Collaborations bagged two notable firsts in the same event. The researchers reported the detection of a coalescing pair of black holes weighing in at 65 and 85 solar masses (see Viewpoint: A Heavyweight Merger). The 142-solar-mass object that resulted from this merger is the only known example of an "intermediate-mass black hole." The heavier of the two progenitors, meanwhile, is the first detected black hole whose mass lies in a range forbidden by current theories of stellar black hole formation. Another out-of-the-ordinary merger reported by the collaborations is that of a "Laurel and Hardy" duo of asymmetric black holes (see Viewpoint: A Lopsided Merger).

\section{Excitement About Nickel-Based Superconductors}

For decades, researchers have tried to find analogs to copper oxide superconductors, or cuprates, but it wasn't until 2019 that they accomplished this goal. "Why the long wait?" teased Mike Norman in his article about the discovery of nickel-based superconductors (see Trend: Entering the Nickel Age of Superconductivity). The synthesis of "nickelates" proved exceptionally difficult, explained Norman, who reviewed the initial interest in the materials and the tricks used to make them. He also wrote that contrasting nickelates with their copper cousins could lead to insights into high-temperature superconductivity-a phenomenon that puzzles physicists to this day (see Synopsis: Nickelates Have Their Own Superconducting Style).

\section{Cosmic Ticker}

Some theorists have proposed that time is not a continuous coordinate but, rather, is determined by the ticking of a physical clock that permeates the Universe-a bit like the Higgs field. Theorists studied how this universal ticker might affect a slower experimental oscillator, such as an atomic clock (see Focus: The
Period of the Universe's Clock). They found that the coupling between the two clocks would desynchronize them and, as a result, no experimental oscillator could maintain a constant period forever. They proposed that measuring the desynching of a pair of atomic clocks might allow confirmation of the existence of the universal oscillator, and they estimated this oscillator's period to be less than $10^{-33}$ seconds.

\section{A Neural Network's Inner Workings}

Neural networks have a reputation as black boxes-systems that solve challenging tasks through mechanisms that are hard to discern. But researchers have found a way to reveal the inner workings of these tools (see Viewpoint: Physics Insights from Neural Networks). By determining the path of data within the layers of a neutral-network-based model, they deciphered how the network processes information. In an example designed to predict the future positions of Mars and the Sun, the researchers found that the neural network solved the problem through a coordinate transformation. Remarkably, this transformation implied that the model switched from a geocentric to a heliocentric worldview, without being told to do so.

\section{Strange Graphene}

A new page unfolded for graphene, as scientists discovered that the 2D material can be a "strange metal"-meaning that its resistance depends on temperature in an unusual way. Evidence for this strange-metal phase was observed in two sheets of graphene twisted at a so-called magic angle with respect to each other (see Viewpoint: Graphene Reveals Its Strange Side). This isn't the first remarkable property of magic-angle graphene, whose superconductivity was the biggest condensed-matter-physics news of 2018. In trying to understand graphene's many facets, theorists are exploring how this strange-metal behavior might be related to quantum chaos and black hole physics.

\section{Worm Churn}

Drunk sludge worms played a starring role in a study of the collective behavior of self-propelled objects (see Focus: Worm Viscosity). The researchers studied fluids containing dense tangles of the wriggling, centimeter-long worms, which can serve as models of active polymers, such as those present in biological cells. The team controlled the worms' activity either by changing temperature or by adding alcohol to the liquid, which causes the worms to cease all motion. Under gentle 
stirring, fluids with active (sober) worms were less viscous than those with inebriated worms. But surprisingly, active-worm fluids became the more viscous ones with more vigorous stirring. 